\title{
A metabolomics-binding network pharmacology study of Zuojin Pill intervention in chronic atrophic gastritis induced by Helicobacter pylori
}

Shihua Wu (D1263588540@qq.com )

Chengdu University of Traditional Chinese Medicine

\section{Xing Chen}

Chengdu University of Traditional Chinese Medicine

Honghong Liu

The fifth medical center of PLA Hospital

\section{Ruilin Wang}

The Fifth Medical Center of PLA Hospital

Jianyu Li

The Fifth Medical Center of PLA General Hospital

Jianxia Wen

Chengdu University of Traditional Chinese Medicine

Tao Yang

Chengdu University of Traditional Chinese Medicine

Ying Wei

Chengdu University of Traditional Chinese Medicine

\section{Sichen Ren}

Chengdu University of Traditional Chinese Medicine

\section{Shizhang Wei}

Chengdu University of Traditional Chinese Medicine

\section{Manyi Jing}

The Flfth Medical Center of PLA General Hospital

Haotian Li

The Fifth Medical Center of PLA General Hospital

\section{Min Wang}

The Fifth Medical Center of PLA General Hospital

Houlin Xia ( 352318327@qq.com )

Chengdu University of Traditional Chinese Medicine

Yanling Zhao ( $\nabla$ 835453882@qq.com )

The Fifth Medical Center of PLA General Hospital 
Research

Keywords: Zuojin Pill, Chronic atrophic gastritis, Helicobacter pylori, Serum metabolomics, Urine metabolomics, Network pharmacology

Posted Date: August 19th, 2020

DOI: https://doi.org/10.21203/rs.3.rs-60895/v1

License: (c) (i) This work is licensed under a Creative Commons Attribution 4.0 International License.

Read Full License 


\section{Abstract}

Background Zuojin Pill (ZJP) is widely used for the treatment of gastrointestinal diseases, while its specific mechanism has not been systematically investigated. The aim of this study was to explore the mechanism of intervention of ZJP in chronic atrophic gastritis (CAG) through metabolomics combined with network pharmacology.

Materials and methods Potential metabolites and possible pathways for ZJP treatment of CAG were explored using a UPLC-Q-TOF/MS-based metabolomics technique. The key targeting mechanism of ZJP for CAG was explored by combining the analysis with network pharmacology.

Results ZJP significantly reduced serum levels of IL-1 $\beta$, IL-6, IL-10 and iNOS, and improved pathological characteristics. Metabolomic results indicated that the therapeutic effect of ZJP was mainly related to ten metabolites, including choline, L-threonine, hydroxypyruvic acid, creatine, taurine, succinic acid, cisaconitic acid, citric acid, succinic acid semialdehyde and uric acid. Pathway analysis showed that the treatment of CAG by ZJP was associated with taurine and hypotaurine metabolism, glyoxylate and dicarboxylate metabolism, glycine, serine and threonine metabolism, glycerophospholipid metabolism, citrate cycle (TCA cycle), alanine, aspartate and glutamate metabolism, butanoate metabolism and purine metabolism. Validation of potential metabolic markers and key targets of network pharmacology by RT-PCR analysis showed that ZJP significantly down-regulated a series of inflammatory markers, such as MAPK1, PKIA, RB1, SCN5A, RXRA, E2F1, PTGS1, IGF2, ADRB1, ADRA1B, PTGS2, and GABRA1.

Conclusion For the first time, a combination of metabolomics and network pharmacology has been used to clarify the therapeutic effects of ZJP on CAG and its relationship to the regulation of multiple metabolic pathways.

\section{Introduction}

Helicobacter pylori (H. pylori) was defined as a type 1 carcinogen by the World Health Organization's International Agency for Research and Cancer (IARC) in 1993, and it is widely accepted that H. pylori infection induces chronic atrophic gastritis (CAG) and even the development of gastric cancer, and several studies have now confirmed this association [1-2]. CAG is a common clinical digestive disorder that is the result of the inflammatory process of $\mathrm{H}$. pylori infection and is commonly characterized by thinning of the mucosal epithelium and atrophy and loss of glands. Although triple and quadruple therapies are commonly used in clinical practice to treat $C A G$, long-term use enhances bacterial resistance and decreases eradication rates and can lead to a number of side effects, such as gastrointestinal reactions and liver dysfunction [3]. Therefore, there is an urgent clinical need for an effective drug with less side effects to treat $\mathrm{H}$. pylori induced CAG.

Zuojin Pill (ZJP) is a famous Chinese herbal formula, first recorded centuries ago in a ancient medical treatise "Danxi New Law". ZJP consists of two traditional Chinese medicines Coptidis Rhizoma (CR) and Euodiae Fructus (EF) according to a weight ratio of $6: 1(\mathrm{w} / \mathrm{w})$ and has been officially listed in the Chinese 
Pharmacopoeia. The main ingredients in CR are alkaloids, including berberine, palmatine, coptisine and epiberberine. Studies have shown that CR has a wide range of pharmacological effects, such as antibacterial, anti-atherosclerotic, antiviral, antioxidant, anti-hepatic steatosis, anti-inflammatory and antitumor effects [4]. EF is the dried near-mature fruit of Euodia rutaecarpa (Juss.) Benth., and the main chemical constituents of EF include alkaloids, and terpenes which have antibacterial, hypotensive and anti-hypoxic pharmacological activities. ZJP has been shown to have anti-inflammatory, anti-ulcer, and anti-acid pharmacological effects, including inhibition of $H$. pylori growth and proliferation [5]. Currently, ZJP is clinically used to treat a variety of gastrointestinal disorders such as gastritis, cholecystitis, and peptic ulcers [6]. Previous studies have shown that ZJP and its constituent active components of herbal CR and EF exhibit multiple pharmacological effects on cancer through multiple molecular mechanism [78]. ZJP plays a gastroprotective role by modulating the NF-KB signaling pathway to regulate inflammatory cytokines and thereby reduce the risk of gastric ulcers [9]. ZJP treats gastrointestinal disorders by restoring the rhythm of the stomach as well as improving gastrointestinal peristalsis [10]. The ethanolic extract of ZJP inhibited inflammation by regulating the expression of iNOS, COX-2, IL-6, IL$1 \beta$, and TNF-a [11]. These studies provide a basis for the therapeutic effect of ZJP on gastric ulcers, gastritis and other clinical inflammatory diseases.

As an important branch of systems biology, metabolomics conducts a systematic research of the relationships between, medicine, disease and metabolites. Metabolomics not only characterizes the overall physiological and pathological state after exogenous stimuli, but also systematically identifies endogenous small molecule metabolites. Modern analytical technologies, including nuclear magnetic resonance (NMR), gas chromatography-mass spectrometry (GC-MS) and liquid chromatography-mass spectrometry (LC-MS) are combined with multivariate data analysis, such as principal component analysis (PCA), partial least-squares discriminate analysis (PLS-DA) and orthogonal projection to latent structures-discriminant analysis (OPLS-DA) [12]. In these methods, ultra-high performance liquid chromatography with quadrupole time-of-flight (UPLC-Q-TOF) has the advantages of high selectivity, resolution and accuracy and is particularly suitable for traditional Chinese medicine (TCM) [13]. Due to the easy collection of blood and urine samples and their close association with diseases, choice of these two biological fluids may be a novel and effective methods for metabolomics research [14]. Serum metabolite profiles are considered to be important indicator of physiology and pathology and can help to understand the interventions, metabolic pathways and mechanisms of disease progression. Urine metabolite profiles is important for the discovery of disease biomarkers, particularly excreted metabolites. Serum samples typically represent low-polarity metabolites, while urine samples consist of high-polarity metabolites. These two metabolite profiles complement each other and reflect overall characteristics, demonstrating the mechanism, intervention and metabolic pathways [15]. Nevertheless, the variety of metabolites and their wide range of concentrations limits the ability to understand disease progression using metabolomics alone, metabolomics analysis of interference with metabolic pathways is still far from completely elucidating the mechanism of TCM. In recent years, network pharmacology has been used to investigate and anticipate mechanisms of drug-target interactions in biological metabolism and related pathways [16]. It provides a systems level approach to understanding the interactions between 
disease features, physiologically active substances and drug targets [17]. A growing number of studies have shown that network pharmacology, as a holistic and effective tool for studying the effects of TCM, can better understand the complexity of TCM and elucidate its underlying mechanisms through understanding its active chemical components and therapeutic targets [18]. In addition, integrated metabolomics and network pharmacology have been successfully used to explore the interactions between TCM and disease targets, which provide important directions for the therapeutic mechanisms of TCM.

In order to elucidate the intervention of ZJP in the treatment of CAG, this study explored the pathological changes and serum biochemical parameters of ZJP through pharmacological experiments based on metabolomics. A rat model of CAG was established by $\mathrm{H}$. pylori, and the success of the model was assessed by a rapid urease kit. UPLC-Q-TOF/MS serum metabolomics and urine metabolomics were used to determine the changes of endogenous metabolites in urine and serum caused by ZJP and to screen relevant targets in combination with network pharmacology techniques to elucidate possible biomarkers and potential metabolic pathways for ZJP treatment of CAG, providing new strategy and feasibility analysis.

\section{Methods}

\section{Reagents}

Interleukin-6 (IL-6, Lot: 202007), interleukin-10 (IL-10, Lot: 202007), interleukin-1ß (IL-1ß, Lot: 202007) and inducible nitric oxide synthasedetection (iNOS, Lot: 202007) ELISA kits were bought from Shanghai MLBIO Biotechnology Co.Ltd (Shanghai, China). RNA-Quick Extraction Kit (No: RN001) was purchased from Beijing Yishan Biotechnology Co.Ltd (Beijing, China).

\section{Preparation of ZJP water extract}

CR (Lot: 18011901) and EF (Lot: 17021602) were purchased from Beijing Lvye Pharmaceutical Co., Ltd. (Beijing, China). Firstly, ZJP was extracted with boiling water (1/10, w/w) two times, $1.0 \mathrm{~h}$ each time. The aqueous extract of ZJP was then dried to powder in a freeze-vacuum drying oven, and the final yield of ZJP was approximately $25.59 \%$.

\section{Bacterial culture and animal handling}

H. pylori strain (strain number: ICDC111001) was supplied by Dr. Jianzhong Zhang (Chinese Disease Control and Prevention Center, Beijing, China). H. pylori strains cultured on blood agar (Thermo Fisher Scientific, China) in a 3-5 day cycle.

170-190g male Sprague-Dawley rats were provided by Beijing Sibeifu Animal Breeding Center [Permission No. SCXK-(Jing) 2016-0002] and fed under standard environment (temperature: $25^{\circ} \mathrm{C} \pm 2^{\circ} \mathrm{C}$, humidity: $55 \% \pm 5 \%, 12: 12 \mathrm{~h}$ light: dark cycle) at the Animal Experimental Center of the Fifth Medical Center of the PLA General Hospital. Thirty-six rats were randomly divided into 6 groups. Rats in the control group were 
gavaged with regular saline, and rats in the other five groups were gavaged with $\mathrm{H}$. pylori suspension $\left(1.5 \times 10^{8}\right.$ colony forming units $\left.(\mathrm{CFU}) / \mathrm{ml}, 1.5 \mathrm{~mL}\right)$ four times a week for 8 weeks. Urine was collected from rats 12 hours after the last treatment for 12 hours. Then, except for the control and model groups, ZJP $(0.63,1.26,2.52 \mathrm{~g} / \mathrm{kg} /$ day $)$ and omeprazole $(1.8 \mathrm{mg} / \mathrm{kg} / \mathrm{day}$, as a positive group) were administered by gavage for 4 weeks. Twelve hours after the last treatment, the rats were executed and stomach and blood were collected. The blood was centrifuged at $3,500 \mathrm{rpm}$ for $15 \mathrm{~m}$ and the serum was separated. The serum and stomach tissues were stored at $-80^{\circ} \mathrm{C}$ for further experiments.

Serum levels of IL-6, IL-10, IL-1 $\beta$ and iNOS were measured according to the kit instructions. Stomach tissues were fixed in $10 \%$ neutral formalin buffer for 24 hours. The fixed gastric tissues were embedded in paraffin wax and then cut into slices using a slicer. Hematoxylin-eosin (HE) was used to highlight gastric sinus injury and inflammatory cell infiltration.

\section{Sample preparation and UPLC-Q-TOF/MS testing}

Mix $600 \mu \mathrm{L}$ of methanol with $200 \mu \mathrm{L}$ of serum. The mixture was allowed to stand at $4^{\circ} \mathrm{C}$ for 20 minutes and then centrifuged at $12,000 \mathrm{rpm}$ for 10 minutes. Finally, the supernatant was aspirated and filtered through a $0.22 \mu \mathrm{m}$ micropore filter. The filtrate was collected and analyzed in the next step, and the urine sample was prepared in the same procedure as the serum sample.

The analysis of serum metabolic profile and urine metabolic profile was performed by Agilent 6550 iFunnel Q-TOF LC/MS (Agilent Technologies, USA) system. On a ZORBOX RRHD $\mathrm{C}_{18}$ analytical column (2.1 mm i.d. $\times 100 \mathrm{~mm}, 1.8 \mu \mathrm{mi}$.d., Agilent Technologies, USA), $4 \mu \mathrm{L}$ of each sample was taken and injected into the system, and samples were separated at $30^{\circ} \mathrm{C}$. Solvent $\otimes$ (water containing $0.1 \%$ formic

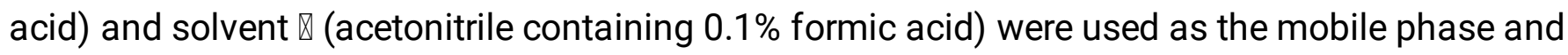
separated at a flow rate of $0.30 \mathrm{~mL} / \mathrm{min}$ for $25 \mathrm{~min}$ with a linear gradient of $100 \%$ over $0-1.0 \mathrm{~min}$, over

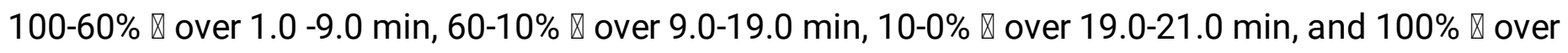
21.0-25.0 min.

Positive and negative mode electrospray source parameters were set as follows: electrospray capillary voltage of $3.5 \mathrm{kV}$ in negative mode and $4 \mathrm{kV}$ in positive mode, mass range of $\mathrm{m} / \mathrm{z} 50-1200$, gas temperature and flow rate were $225^{\circ} \mathrm{C}$ and $13 \mathrm{~L} / \mathrm{min}$, atomizer setting of $20 \mathrm{psi}$, sheath gas temperature and flow rate were $275^{\circ} \mathrm{C}$ and $12 \mathrm{~L} /$ min with a nozzle voltage of $2000 \mathrm{~V}$ in both positive and negative ion mode.

\section{Data extraction and multivariate analysis}

Data were extracted, peaks detection and comparison performed by using MassHunter Profinder software (Agilent, CA, USA). Full scan mode was applied to the mass range $\mathrm{m} / \mathrm{z} 80-1000$ and the initial and final retention times for data collection were set. Data were standardized using the MetaboAnalyst website (https://www.metaboanalyst.ca) and then analyzed by principal component analysis (PCA) and 
orthogonal-partial least squares-discriminant analysis (OPLS-DA) using SIMCA-P 14.1 software (Umetrics, Umea, Sweden).

\section{Potential biomarker identification and pathway enrichment analysis}

Biomarkers were identified by metabolic differential metabolite screening. In the OPLS-DA analysis, the screening conditions for differential metabolites were set to VIP $>1.0, \mid p$ (corr) $\mid \geq 0.58, P<0.05$, and the obtained metabolites were identified as potential biomarkers [19]. Metabolites were identified based on the precise molecular weight of the Human Metabolome Database (HMDB) (molecular weight error $<20$ $\mathrm{ppm}$ ). The identified compounds were resubmitted to MetaboAnalyst to enrich for potential signaling pathways.

\section{ZJP drug target identification and network pharmacology techniques}

The chemical composition and associated targets of CR and EF were collected in the TCMSP database (https://tcmspw.com/tcmsp.php). Potential metabolite-associated protein targets were collected via the MBROLE 2.0 database (http://csbg.cnb.csic.es/mbrole2/index.php). The Genecards database (https://www.genecards.org/) is used to collect CAG-associated protein targets. Uniprot ID was used to convert different types of protein IDs, and then a network of component-metabolite-target interactions was established using protein interaction information. Finally, the component-metabolite-target interaction network was visualized and analyzed using Cytoscape 3.7.1 software.

Real-time polymerase chain reaction (RT-PCR) detection

Total RNA was extracted from gastric tissues using the RNA-Quick Extraction Kit and the total RNA was reverse transcribed to cDNA using the PrimerScript RT kit (Promega, Madison, USA). The CDNA was subsequently amplified by PCR by ABI Step One Plus. The CDNA was subsequently PCR amplified by ABI Step One Plus. The data were analyzed by the $2^{-\triangle \Delta C T}$ method, and the list of primers is shown in Table 1.

\section{Statistical Analysis}

All data were presented as mean \pm standard deviation (SD) and analyzed using the SPSS software program (version 25.0; SPSS Inc., Chicago, IL, USA). The differences were considered to be statistically significant when $P<0.05$ and highly significant when $P<0.01$.

\section{Results}

\section{ZJP reduces $\mathrm{H}$. pylori-induced CAG pathological damage and serum inflammatory factors}

As shown in Figure 1, the control group rats had normal gastric histology, and the model group rats showed pathological features of CAG, such as epithelial rupture of the gastric mucosa, atrophy and misarrangement of glands, accompanied by inflammatory cell infiltration. ZJP administration was effective in improving gastric mucosal lesions, glandular loss, and reducing inflammatory infiltration, 
especially in the $2.52 \mathrm{~g} / \mathrm{kg}$ ZJP group. Moreover, the serum levels of IL-1 $\beta$, IL-6, IL-10 and iNOS were significantly increased in the model group rats compared with the control group $(P<0.01)$. serum concentrations of IL-1 $\beta$, IL-6, IL-10 and iNOS were decreased after ZJP administration. IL-1 $\beta$, IL-6, IL-10, and iNOS levels were significantly reduced after administration of $2.52 \mathrm{~g} / \mathrm{kg} Z \mathrm{JP}$ compared to the model group $(P<0.01)$.

\section{Multivariate data analysis}

Two analytical methods, PCA and OPLS-DA, are powerful statistical tools that provide insight into the separation between different groups based on the results of different spectra [20]. After Pareto normalization of data from serum and urine samples from the control group, model group and $2.52 \mathrm{~g} / \mathrm{kg}$ ZJP group, the three groups were distinguished by PCA using SIMCA-P 12.0 software. As shown in Figure 3 , there was a good separation between the control group, model group and $2.52 \mathrm{~g} / \mathrm{kg}$ ZJP group in both $\mathrm{ESI}+$ and ESI- modes, indicating a significant difference in the metabolic distribution of the three groups. As shown in Figure 3B, D, there was a clear separation between the serum and urine samples of the control group and model group in ESI- mode, and the $2.52 \mathrm{~g} / \mathrm{kg}$ ZJP group was located between the control group and model group, indicating that ZJP was regulating the metabolically abnormal rats to a normal state. The ESI+ model has a poor trend in the $2.52 \mathrm{~g} / \mathrm{kg}$ ZJP group therefore further analysis is required.

OPLS-DA models were then developed for the ESI+ and ESI- modes of serum and urine samples. Given that OPLS-DA was used to screen for differentially expressed metabolites between the two groups [21], we identified the differential metabolites under OPLS-DA analysis for the control group and model group, and for the model group and $2.52 \mathrm{~g} / \mathrm{kg}$ ZJP group, respectively. As shown in Figure 4 and 5 , in the OPLSDA mode established from the serum and urine data of the control group and model group for ESI+ and ESI- modes could explain the $0.999,0.993,0.991$ and 0.999 variance of the response variable $\left(R^{2} Y\right)$ and the cumulative explained variance for modeling in cross-validations $\left(Q^{2}\right)$ were $0.975,0.938,0.857$ and 0.92, respectively. For serum and urine data of the model group vs $2.52 \mathrm{~g} / \mathrm{kg} \mathrm{ZJP}$ group in ESI+ and ESImodes, $R^{2} Y$ values were $0.998,0.987,0.98$ and 0.989 with $Q^{2} 0.979,0.81,0.774$ and 0.91 , individually. These parameters indicate that the models have good explanatory and predictive capabilities. The S-plot was used to investigate the inherent clustering variables. Variables with an average VIP value above 1 and an absolute $\mathrm{P}$ (corr) value above 0.58 in the corresponding $\mathrm{S}$ plot can be considered as potential biomarkers. The S-curve plots show the apparent differences between the endogenous metabolites in the control group, model group and $2.52 \mathrm{~g} / \mathrm{kg}$ ZJP group. The variables located at the ends of the S-curve made an important contribution to the separation of the two groups. The predictive power of the established OPLS-DA model is then verified by a permutation test $(n=100)$. The results showed that the $R^{2}$ and $Q^{2}$ values of serum and urine data were lower than the permutation tests, indicating the superior fit and better predictive ability of the OPLS-DA model.

\section{Identification of potential metabolites in ZJP treatment}


For more accurate identification, potential metabolites were selected based on the principles of $|\mathrm{VIP}|>1$ and $\mid \mathrm{P}$ (corr) $\mid \geq 0.58$ in the S-plots extracted between the control group and model group, model group and $2.52 \mathrm{~g} / \mathrm{kg}$ ZJP group. At this threshold, variables with significant differences were screened using ANOVA analysis and t-test for multivariate and univariate analyses, respectively. Matching the $\mathrm{m} / \mathrm{z}$ of metabolite candidates to online databases, including the HMDB database and the Metaboanalyst database, identifies candidates with significant changes as biomarkers. The mass tolerance value and the exact mass of the measured $\mathrm{m} / \mathrm{z}$ are defined as less than $20 \mathrm{ppm}$.

For serum samples, a total of six endogenous metabolites were screened in ESI+ mode and ESI- mode (see Table 2 for details). For urine samples, four endogenous metabolites were identified based on the above procedure (please see Table 2). These ten potential biomarkers are choline (C00114), L-threonine (C00188), hydroxypyruvic acid (C00168), creatine (C00300), taurine (C00245), succinic acid (C00042), cis-aconitic acid (C00417), citric acid (C00158), succinic acid semialdehyde (C00232) and uric acid (C00366). The levels of L-threonine, hydroxypyruvic acid, taurine, succinic acid and cis-aconitic acid were significantly lower in the model group compared to the control group $(p<0.05$ and $p<0.01)$. In addition, levels of choline, creatine, citric acid, succinic acid semialdehyde and uric acid were significantly higher in the model group compared to the control group $(p<0.01)$. The mean peak area of these metabolites tended to normalize in the $2.52 \mathrm{~g} / \mathrm{kg}$ ZJP group compared with the model group $(P<0.05$ and $p<0.01)$, showing good therapeutic efficacy. The mean peak area of these metabolites is shown in Figure 6 to visualize the effects of ZJP. Alterations in these parameters indicate that metabolic abnormalities occur in control group rats following gastric mucosal inflammatory injury and that metabolic markers return to normal levels after ZJP treatment.

\section{Metabolic pathway analysis}

To explore the potential mechanism of ZJP's effect on CAG, potential metabolites identified from serum and urine samples were introduced into MetaboAnalyst to construct metabolic pathways, and twentyeight metabolic pathways were obtained. Of these twenty-eight metabolic pathways, seven of them play an important role, including Taurine and hypotaurine metabolism, Glyoxylate and dicarboxylate metabolism, Glycine, serine and threonine metabolism, Glycerophospholipid metabolism, Citrate cycle (TCA cycle), Alanine, aspartate and glutamate metabolism, Butanoate metabolism and Purine metabolism. The match status, $P$ value, $-\log (P)$ and impact of each pathway are listed in Table 3.

\section{Network Pharmacology}

To visualize the interactions between potential metabolites, disease targets and ZJP components, components and potential CAG disease targets were collected and combined with potential metabolites via the TCMSP database and Genecards database to construct a potential metabolite-CAG targetcomponents network, as shown in Figure 8. MBROLE was used to extend this potential metabolite to related metabolic pathways to further explore the relationship between metabolite-associated proteins and disease targets. Information of the 12 proteins of relevance in compounds-disease targets- 
metabolites is presented in Table 4, which are directly regulated by 124 chemical components and collected from ZJP (Figure 8B).

\section{The effect of ZJP on the mRNA expression in gastric tissue of CAG rats}

CAG is a critical step in the development of gastric cancer, and with increased inflammation comes a progressively higher chance of cancer. A large amount of clinical evidence suggests that ZJP inhibits inflammatory lesions including gastric mucosal injury, neutrophil infiltration and intestinal epithelial chemosis in CAG patients. Thus, inflammation related genes MAPK1, PKIA, RB1, SCN5A, RXRA, E2F1, PTGS1, IGF2, ADRB1, ADRA1B, PTGS2, and GABRA1 may play a more important role in ZJP treatment of CAG. To verify the validity of the network pharmacology predictions, RT-PCR was used to detect the mRNA expression of these genes in gastric tissues. As shown in Figure 9, the relative mRNA expression levels of MAPK1, PKIA, RB1, SCN5A, RXRA, E2F1, PTGS1, IGF2, ADRB1, ADRA1B, PTGS2, and GABRA1 in the model group were significantly elevated $(P<0.01)$. The expression levels of the above genes in the ZJP groups were significantly decreased $(P<0.05$ and $P<0.01)$, indicating the effectiveness of ZJP in treating CAG through anti-inflammation.

\section{Discussion}

CAG is a common clinical disorder of the gastrointestinal tract and is considered to be a precancerous condition of gastric cancer. Current conventional treatments for CAG are mainly focus on symptomatic treatment, however, symptomatic treatment is often associated with some degree of side effects, poor efficacy and high relapse rates [22]. Metabolomics combined with network pharmacology can effectively overcome these problems by extensively mining data to identify targets of metabolism and disease [23]. Metabolomics analyzes small molecule levels in serum and urine to explore compounds with significant abnormalities and reveal mechanisms of disease development. Network pharmacology reveals the molecular mechanisms of drug treatment for diseases by determining the interactions between chemical components and disease protein targets. Therefore, in this study, rat serum metabolomics and urine metabolomics based on the UPLC-Q-TOF/MS method combined with network pharmacology were used to elucidate the therapeutic mechanism of ZJP on CAG, which also provides a molecular level exploration of the ZJP mechanism.

H. pylori is the most commonly infected bacterium worldwide and is a significant risk factor for the development of gastric cancer. Numerous studies have been published showing that $\mathrm{H}$. pylori can colonize and grow in an extremely acidic gastric environment, establishing a persistent infection and decreasing host immune function, leading to the development of gastritis and gastric cancer [24]. Currently, H. pylori is widely used to establish rodent models of CAG [25]. Therefore, in the present study, the CAG rat model was established by gavage of $\mathrm{H}$. pylori. HE staining confirmed that ZJP was effective in reducing gastric mucosal damage and maintaining epithelial structural integrity, and improving monocyte and lymphocyte infiltration. Serum biochemical parameters showed that ZJP significantly reduced serum levels of IL-1 $\beta$, IL-6, IL-10 and iNOS, and restored the integrity of gastric mucosa and 
effectively reduced the expression of inflammatory factors. The above results confirm that ZJP shows a therapeutic effect on CAG, which is consistent with clinical efficacy.

Next the changes in serum metabolomic and urine metabolomic characteristics of ZJP in CAG treatment were identified. ZJP protects the normal structure of the gastric mucosa by restoring potential metabolites to normal levels. The screened biomarkers can be effective targets for disease diagnosis, treatment, and prevention [26]. The PCA model revealed significant differences in metabolite distribution between the control group, model group and $2.52 \mathrm{~g} / \mathrm{kg}$ ZJP group. The distribution of potential metabolic biomarkers was identified in the OPLS-DA model. There was a significant separation of biomarkers between the control group and model group, indicating a significant effect of H. pylori on the serum metabolomic profile and the urine metabolomic profile. There were also significant differences between the $2.52 \mathrm{~g} / \mathrm{kg}$ ZJP group and model group, suggesting a therapeutic effect of ZJP on CAG. Finally, six differential metabolites were screened in serum samples and four metabolic differentials in urine samples to reveal the regulatory mechanism of ZJP in the treatment of CAG. These metabolites interact and regulate the disease process in different ways. The results suggest that the occurrence and development of CAG is regulated by changes in many physiologically and pathologically relevant biomarkers, most of which in the organism are mutually influential. ZJP restores normal levels of expression by modulating these ten potential biomarkers, suggesting that ZJP can treat H. pylori-induced CAG through multiple targets and multiple pathways.

In total, ten differential metabolites were identified and were involved in seven major metabolic pathways. These metabolites involve seven major metabolic pathways, including taurine and hypotaurine metabolism, glyoxylate and dicarboxylate metabolism, glycine, serine and threonine metabolism, glycerophospholipid metabolism, TCA cycle, alanine, aspartate and glutamate metabolism, butanoate metabolism and purine metabolism. Taurine and hypotaurine metabolism is important metabolic pathways. Taurine is involved in multiple biological pathways and has a variety of pharmacological activities, such as eye and brain development, immune function, osmoregulation, as well as antioxidant and anti-inflammatory activities. Previous studies have shown that it is protective against oxidative stress-induced gastrointestinal injury [27]. Taurine is one of the most abundant free amino acids in animal tissues and plays an important role in a variety of important physiological processes.

Upregulation of taurine in serum by ZJP treatment may be a marker of inflammatory response. Elevated amino acid levels are frequently observed in patients with gastritis, which helps to distinguish CAG patients from normal people [28]. In addition, metabolic pathway analysis identified several amino acidrelated pathways in $C A G$, including the metabolism of glycine, serine, and threonine and the metabolism of alanine, aspartate, and glutamate. The results of the present study showed that the expression of choline, creatine, and succinic acid semialdehyde associated with amino acid metabolism was elevated and L-threonine expression was decreased in the model group, and this trend was ameliorated by ZJP administration. The involvement of glyoxylate and dicarboxylate metabolism further supports the involvement of ZJP in energy metabolism. Gastric wall cells contain a large number of mitochondria [29]. Mitochondria provide energy to the cells through oxidative phosphorylation, which plays an important role in maintaining the steady state and function of the gastric mucosa. ZJP further elevates

Page 11/28 
hydroxypyruvate expression by restoring energy metabolism. Purines are essential components of nucleotides in cell proliferation, thus impaired purine metabolism is associated with inflammation and the development of cancer [30]. The expression of uric acid was elevated in CAG rats, which is consistent with what has been reported in the research [31]. The TCA cycle is the final metabolic pathway for carbohydrates, lipids, and amino acids and is the most important metabolic pathway for energy supply. Succinic acid and cis-aconitic acid belong to the TCA cycle and mediate the formation and metabolism of intracellular ATP [32]. The citric acid is a key intermediate of the TCA cycle [33]. The results showed that succinic acid and cis-aconitic acid contents were decreased and citric acid contents were significantly increased in CAG rats. These results indicate the importance of the TCA cycle in the pathological process of CAG.

In an attempt to further understand the mechanism of H. pylori-induced CAG by ZJP treatment and the association between chemical composition and metabolites, we established a "compounds-disease targets-metabolites" interaction network by combining network pharmacology. The results showed that 124 chemical components originating from ZJP acted directly with 12 potential protein targets, suggesting the advantages of multiple components in ZJP in regulating endogenous metabolic changes in the body, showing the advantages of ZJP in the clinical treatment of CAG. Twelve targets were strongly associated with inflammation, including MAPK1, PTGS2, PTGS1, RXRA, ADRA1B, RB1, ADRB1, E2F1, SCN5A, PKIAIGF2, and GABRA1 [34-37]. Therefore, to verify the accuracy of the network pharmacology predictions and to further elucidate the potential mechanism of ZJP, we further tested the expression of these genes in gastric tissues of CAG rats. The expression of MAPK1, PTGS2, PTGS1, RXRA, ADRA1B, RB1, ADRB1, E2F1, SCN5A, PKIAIGF2, and GABRA1 was significantly elevated in the CAG model group. However, ZJP significantly reduced the relative mRNA expression of these genes. These results suggest that ZJP may play a role in the treatment of CAG by further ameliorating gastric mucosal inflammatory lesions through anti-inflammation.

\section{Conclusion}

This study systematically investigated the efficacy of ZJP for the treatment of $\mathrm{H}$. pylori-induced CAG and its molecular mechanisms through a combination of metabolomics and network pharmacology. This study can lay a foundation for further understanding of the interactions between components and targets of TCM and provide a theoretical basis for guiding TCM clinical practice.

\section{Declarations}

\section{Acknowledgements}

Not applicable.

\section{Authors' contributions}


Design of the study: Honghong Liu, Ruilin Wang and Jianyu Li; data collection and analysis: Shihua Wu, Xing Chen and Min Wang; drafting the manuscript: Jianxia Wen, Tao Yang, Ying Wei, Sichen Ren, Shizhang Wei, Manyi Jing and Haotian Li; supervising and providing Funding acquisition: HouLin Xia, Yanling Zhao. All authors participated in amending the manuscript before submission of the mutually agreed final version.

\section{Funding}

This study was supported by the National key R \& D Program of China [No. 2018YFC1704500].

\section{Availability of data and materials}

The datasets used and/or analyzed in the present study are available from the corresponding author on reasonable request.

\section{Ethics approval and consent to participate}

All animal experiments were performed under the approval of the Ethics Committee of the Ethics of Animal Experiments of the Fifth Medical Center of PLA General Hospital (Approval ID: IACUC-2018-010).

\section{Consent for publication}

All authors agree to publish this paper.

\section{Competing interests}

The authors declare that they have no competing interests.

\section{Author details}

${ }^{1}$ College of pharmacy, Chengdu University of Traditional Chinese Medicine, Chengdu, China. ${ }^{2}$ Department of Pharmacy, The Fifth Medical Center of PLA General Hospital, Beijing, China. ${ }^{3}$ Integrative Medical Center, The Fifth Medical Center of PLA General Hospital, Beijing, China.

\section{Abbreviations}

ZJP: Zuojin Pill; CAG: Chronic atrophic gastritis; Helicobacter pylori: H. pylori; TCA cycle: citrate cycle; IARC: International Agency for Research and Cancer; CR: Coptidis Rhizoma; EF: Euodiae Fructus; NMR: nuclear magnetic resonance; GC-MS: gas chromatography-mass spectrometry; LC-MS: liquid chromatography-mass spectrometry; PCA: principal component analysis: PLS-DA: partial least-squares discriminate analysis; OPLS-DA: orthogonal projection to latent structures-discriminant analysis; UPLC-QTOF: ultra-high performance liquid chromatography with quadrupole time-of-flight; TCM: traditional Chinese medicine. 


\section{References}

[1] An international association between Helicobacter pylori infection and gastric cancer. The EUROGAST Study Group [published correction appears in Lancet 1993 Jun 26;341(8861):1668]. Lancet. 1993;341(8857):1359-1362.

[2] Uemura N, Okamoto S, Yamamoto S, et al. Helicobacter pylori infection and the development of gastric cancer. N Engl J Med. 2001;345(11):784-789.

[3] Yoon H, Kim N, Lee BH, et al. Moxifloxacin-containing triple therapy as second-line treatment for Helicobacter pylori infection: effect of treatment duration and antibiotic resistance on the eradication rate. Helicobacter. 2009;14(5):77-85.

[4] Ma BL, Ma YM. Pharmacokinetic properties, potential herb-drug interactions and acute toxicity of oral Rhizoma coptidis alkaloids. Expert Opin Drug Metab Toxicol. 2013;9(1):51-61.

[5] Chen YF, Chen WW, Li RL. Effect of Zuojin wan and retro-zuojin wan on inflammatory and protection factors of chills and fever gastric mucosa injury. Chin J Integr Tradit West Med Dig. 2003;11:133-135.

[6] Cheng DH, Zhao YL, Yang HB. Effect of zuojin pill and fanzuojin pill on the growth metabolism of enterobacteria by microcalorimetry. Zhongguo Zhong Xi Yi Jie He Za Zhi. 2011;31:209-212.

[7] Chou ST, Hsiang CY, Lo HY, et al. Exploration of anti-cancer effects and mechanisms of Zuo-JinWan and its alkaloid components in vitro and in orthotopic HepG2 xenograft immunocompetent mice. BMC Complement Altern Med. 2017;17(1):121. Published 2017 Feb 20.

[8] Pan J, Xu Y, Song H, Zhou X, Yao Z, Ji G. Extracts of Zuo Jin Wan, a traditional Chinese medicine, phenocopies 5-HTR1D antagonist in attenuating Wnt/ $\beta$-catenin signaling in colorectal cancer cells. BMC Complement Altern Med. 2017;17(1):506. Published 2017 Nov 28.

[9] Wang J, Zhang T, Zhu L, Ma C, Wang S. Anti-ulcerogenic effect of Zuojin Pill against ethanolinduced acute gastric lesion in animal models. J Ethnopharmacol. 2015;173:459-467.

[10] Wang T, Yan YF, Yang L, et al. Effects of Zuojin pill on depressive behavior and gastrointestinal function in rats with chronic unpredictable mild stress: Role of the brain-gut axis. J Ethnopharmacol. 2020;254:112713.

[11] Wang QS, Cui YL, Dong TJ, Zhang XF, Lin KM. Ethanol extract from a Chinese herbal formula, "Zuojin Pill", inhibit the expression of inflammatory mediators in lipopolysaccharide-stimulated RAW 264.7 mouse macrophages. J Ethnopharmacol. 2012;141(1):377-385.

[12] Zira AN, Theocharis SE, Mitropoulos D, Migdalis V, Mikros E. (1)H NMR metabonomic analysis in renal cell carcinoma: a possible diagnostic tool. J Proteome Res. 2010;9(8):4038-4044. 
[13] Wu L, Hao H, Wang G. LC/MS based tools and strategies on qualitative and quantitative analysis of herbal components in complex matrixes. Curr Drug Metab. 2012;13(9):1251-1265.

[14] Dunn WB, Broadhurst D, Begley P, et al. Procedures for large-scale metabolic profiling of serum and plasma using gas chromatography and liquid chromatography coupled to mass spectrometry. Nat Protoc. 2011;6(7):1060-1083. Published 2011 Jun 30.

[15] Yang QJ, Zhao JR, Hao J, et al. Serum and urine metabolomics study reveals a distinct diagnostic model for cancer cachexia. J Cachexia Sarcopenia Muscle. 2018;9(1):71-85.

[16] Ren Jun-Ling, Dong Hui, Han Ying et al. Network pharmacology combined with metabolomics approach to investigate the protective role and detoxification mechanism of Yunnan Baiyao formulation. [J] .Phytomedicine, 2020, 77: 153266.

[17] Ning K., Zhao X., Poetsch A., Chen W.-H., Yang J. Computational molecular networks and network pharmacology. BioMed Research International. 2017;2017:1.

[18] Barabási A. L., Oltvai Z. N. Network biology: understanding the cell's functional organization. Nature Reviews Genetics. 2004;5(2):101-113. doi: 10.1038/nrg1272.

[19] Blasco H, Błaszczyński J, Billaut JC, et al. Comparative analysis of targeted metabolomics: dominance-based rough set approach versus orthogonal partial least square-discriminant analysis. J Biomed Inform. 2015;53:291-299.

[20] Worley B, Powers R. PCA as a practical indicator of OPLS-DA model reliability. Curr Metabolomics. 2016;4(2):97-103.

[21] Wei S, Qian L, Niu M, et al. The Modulatory Properties of Li-Ru-Kang Treatment on Hyperplasia of Mammary Glands Using an Integrated Approach. Front Pharmacol. 2018;9:651. Published 2018 Jun 19.

[22] Park YH, Kim N. Review of atrophic gastritis and intestinal metaplasia as a premalignant lesion of gastric cancer. J Cancer Prev. 2015;20(1):25-40.

[23] Zhang Y, Gao J, Zhang Q, et al. Interpretation of Euphorbia Kansui Stir-Fried with Vinegar Treating Malignant Ascites by a UPLC-Q-TOF/MS Based Rat Serum and Urine Metabolomics Strategy Coupled with Network Pharmacology. Molecules. 2018;23(12):3246.

[24] Camilo V, Sugiyama T, Touati E. Pathogenesis of Helicobacter pylori infection. Helicobacter. 2017;22 Suppl 1:10.1111/hel.12405.

[25] Wei X, Feng XP, Wang LY, et al. Improved method for inducing chronic atrophic gastritis in mice. World J Gastrointest Oncol. 2019;11(12):1115-1125. 
[26] Jiang W, Si L, Li P, et al. Serum metabonomics study on antidiabetic effects of fenugreek flavonoids in streptozotocin-induced rats. J Chromatogr B Analyt Technol Biomed Life Sci. 2018;1092:466-472.

[27] Zhou J, Yao N, Wang S, et al. Fructus Gardeniae-induced gastrointestinal injury was associated with the inflammatory response mediated by the disturbance of vitamin B6, phenylalanine, arachidonic acid, taurine and hypotaurine metabolism. J Ethnopharmacol. 2019;235:47-55.

[28] Chan AW, Gill RS, Schiller D, Sawyer MB. Potential role of metabolomics in diagnosis and surveillance of gastric cancer. World J Gastroenterol. 2014;20(36):12874-12882.

[29] Duman JG, Pathak NJ, Ladinsky MS, McDonald KL, Forte JG. Three-dimensional reconstruction of cytoplasmic membrane networks in parietal cells. J Cell Sci. 2002;115(Pt 6):1251-1258.

[30] Yin J, Ren W, Huang X, Deng J, Li T, Yin Y. Potential Mechanisms Connecting Purine Metabolism and Cancer Therapy. Front Immunol. 2018;9:1697. Published 2018 Jul 30.

[31] Zhang H, Fu P, Ke B, et al. Metabolomic analysis of biochemical changes in the plasma and urine of collagen-induced arthritis in rats after treatment with Huang-Lian-Jie-Du-Tang. J Ethnopharmacol. 2014;154(1):55-64.

[32] Shiratori R, Furuichi K, Yamaguchi M, et al. Glycolytic suppression dramatically changes the intracellular metabolic profile of multiple cancer cell lines in a mitochondrial metabolism-dependent manner. Sci Rep. 2019;9(1):18699. Published 2019 Dec 10.

[33] Chen, M., Liu, R., Weng, Y., 2012. Discovery and enlightenment of the tricarboxylic acid cycle.

Med. philo 33 (1), 71-73.

[34] Bjarnason I, Scarpignato C, Holmgren E, Olszewski M, Rainsford KD, Lanas A. Mechanisms of Damage to the Gastrointestinal Tract From Nonsteroidal Anti-Inflammatory Drugs. Gastroenterology. 2018;154(3):500-514.

[35] McAbee J, Li Q, Yu H, Kirkwood KL. Sexual dimorphism in periapical inflammation and bone loss from mitogen-activated protein kinase phosphatase-1 deficient mice. J Endod. 2012;38(8):1097-1100.

[36] Mukwaya A, Lennikov A, Xeroudaki M, et al. Time-dependent LXR/RXR pathway modulation characterizes capillary remodeling in inflammatory corneal neovascularization. Angiogenesis. 2018;21(2):395-413.

[37] White CR, Dungan M, Carrithers MD. Activation of human macrophage sodium channels regulates RNA processing to increase expression of the DNA repair protein PPP1R10. Immunobiology. 2019;224(1):80-93. 


\section{Tables}

Table 1 Primers used for real-time PCR

\begin{tabular}{|lll|}
\hline Primers & Sequence-Forward & Sequence-Reverse \\
\hline PTGS1 & TCTGGACCTGGCTTCGGAGTTC & TGGACCGCACCGTGAGTACC \\
\hline PTGS2 & AGGTCATCGGTGGAGAGGTGTATC & CGGCACCAGACCAAAGACTTCC \\
\hline MAPK1 & TGAAGACACAGCACCTCAGCAATG & GGTGTTCAGCAGGAGGTTGGAAG \\
\hline GABRA1 & AGTGCCAGAAATTCCCTCCCAAAG & CAATCAGAGCCGAGAACACGAAGG \\
\hline IGF2 & CAGCCGTGGCATCGTGGAAG & CGGGGTATCTGGGGAAGTCGTC \\
\hline PKIA & TTCCTCTGCAAGTGGCAACAGC & TGCTTCTCCCTGGGCTTCGC \\
\hline SCN5A & GGAAGGTCTGGTGGCGATTGC & TTCCGCTCCTCCAGGTAGATGTC \\
\hline E2F1 & TGGTGGCTGCTGACTCACTCC & TGGGGTGGGGAAAGGCTGATG \\
\hline ADRB1 & CTCATCGTGCTGCTCATCGTAGTG & GCCAGGGACATGATGAAGAGGTTG \\
\hline RB1 & CAAGCAGCCTCAGCCTTCCATAC & TGTGTTCTGGCTCTGGGTGGTC \\
\hline ADRA1B & ATCGTGGGCAACATCTTGGTCATC & ACTCAACAGCAGGTCAGCAATGG \\
\hline RXRA & ACACTGGGCTTCGGGACTGG & GGAACCTTGAGGACGCCATTGAG \\
\hline B-Actin & CCCGCGAGTACAACCTTCTTG & TCATCCATGGCGAACTGGTGG \\
\hline
\end{tabular}

Table 2 Identification of serum and urinary metabolites in different groups. 


\begin{tabular}{|c|c|c|c|c|c|c|}
\hline No. & $\mathrm{RT}(\min )$ & mass & Metabolites & Formula & KEGG & Pathway \\
\hline 1 & 17.65 & 104.1069 & Choline & $\mathrm{C}_{5} \mathrm{H}_{14} \mathrm{NO}$ & C00114 & $\begin{array}{l}\text { Glycine, serine and } \\
\text { threonine metabolism }\end{array}$ \\
\hline 2 & 2.33 & 120.0658 & L-Threonine & $\mathrm{C}_{4} \mathrm{H}_{9} \mathrm{NO}_{3}$ & C00188 & $\begin{array}{l}\text { Glycine, serine and } \\
\text { threonine metabolism }\end{array}$ \\
\hline 3 & 7.58 & 126.9998 & $\begin{array}{l}\text { Hydroxypyruvic } \\
\text { acid }\end{array}$ & $\mathrm{C}_{3} \mathrm{H}_{4} \mathrm{O}_{4}$ & C00168 & $\begin{array}{l}\text { Glyoxylate and } \\
\text { dicarboxylate } \\
\text { metabolism }\end{array}$ \\
\hline 4 & 0.90 & 132.0771 & Creatine & $\mathrm{C}_{4} \mathrm{H}_{9} \mathrm{~N}_{3} \mathrm{O}_{2}$ & $\mathrm{C00300}$ & $\begin{array}{l}\text { Glycine, serine and } \\
\text { threonine metabolism }\end{array}$ \\
\hline 5 & 0.89 & 124.0073 & Taurine & $\mathrm{C}_{2} \mathrm{H}_{7} \mathrm{NO}_{3} \mathrm{~S}$ & $\mathrm{C} 00245$ & $\begin{array}{l}\text { Taurine and } \\
\text { hypotaurine } \\
\text { metabolism }\end{array}$ \\
\hline 6 & 14.66 & 119.034 & Succinic acid & $\mathrm{C}_{4} \mathrm{H}_{6} \mathrm{O}_{4}$ & C00042 & TCA cycle \\
\hline 7 & 0.78 & 173.0087 & cis-Aconitic acid & $\mathrm{C}_{6} \mathrm{H}_{6} \mathrm{O}_{6}$ & C00417 & TCA cycle \\
\hline 8 & 1.22 & 215.0165 & Citric acid & $\mathrm{C}_{6} \mathrm{H}_{8} \mathrm{O}_{7}$ & C00158 & TCA cycle \\
\hline 9 & 0.79 & 101.0239 & $\begin{array}{l}\text { Succinic acid } \\
\text { semialdehyde }\end{array}$ & $\mathrm{C}_{4} \mathrm{H}_{6} \mathrm{O}_{3}$ & C00232 & $\begin{array}{l}\text { Alanine, aspartate and } \\
\text { glutamate metabolism }\end{array}$ \\
\hline 10 & 0.87 & 167.0202 & Uric acid & $\mathrm{C}_{5} \mathrm{H}_{4} \mathrm{~N}_{4} \mathrm{O}_{3}$ & C00366 & Purine metabolism \\
\hline
\end{tabular}

Table 3 Results of integrating enrichment analysis of biomarkers with MetaboAnalyst 


\begin{tabular}{|c|c|c|c|c|c|c|}
\hline & No. & Pathway name & $\begin{array}{l}\text { Match } \\
\text { status }\end{array}$ & $P$ value & $-\log (p)$ & Impact \\
\hline \multirow[t]{5}{*}{ serum } & 1 & $\begin{array}{l}\text { Taurine and hypotaurine } \\
\text { metabolism }\end{array}$ & $1 / 8$ & 0.05192 & 2.9581 & 0.42857 \\
\hline & 2 & $\begin{array}{l}\text { Glyoxylate and dicarboxylate } \\
\text { metabolism }\end{array}$ & $1 / 32$ & 0.19345 & 1.6427 & 0.21958 \\
\hline & 3 & $\begin{array}{l}\text { Glycine, serine and threonine } \\
\text { metabolism }\end{array}$ & $4 / 34$ & $4.1098 \times 10^{-5}$ & 10.1 & 0.06662 \\
\hline & 4 & Glycerophospholipid metabolism & $2 / 36$ & 0.022085 & 3.8128 & 0.04318 \\
\hline & 5 & Citrate cycle (TCA cycle) & $1 / 20$ & 0.12526 & 2.0774 & 0.03273 \\
\hline \multirow[t]{6}{*}{ urine } & 6 & Citrate cycle (TCA cycle) & $2 / 20$ & 0.0085481 & 4.762 & 0.14041 \\
\hline & 7 & Glycerophospholipid metabolism & $1 / 36$ & 0.23395 & 1.4527 & 0.09446 \\
\hline & 8 & $\begin{array}{l}\text { Glyoxylate and dicarboxylate } \\
\text { metabolism }\end{array}$ & $2 / 32$ & 0.021272 & 3.8503 & 0.05556 \\
\hline & 9 & $\begin{array}{l}\text { Alanine, aspartate and glutamate } \\
\text { metabolism }\end{array}$ & $2 / 28$ & 0.016472 & 4.1061 & 0.04808 \\
\hline & 10 & Butanoate metabolism & $1 / 15$ & 0.1044 & 0.1044 & 0.03175 \\
\hline & 11 & Purine metabolism & $1 / 66$ & 0.38959 & 0.94266 & 0.94266 \\
\hline
\end{tabular}

Table 4 Participating in the "Component-Target-Potential Metabolite" interactive network for ZJP treatment of CAG 


\begin{tabular}{|lll|}
\hline Uniprot ID & Gene name & Protein name \\
\hline P28482 & MAPK1 & Mitogen-activated protein kinase 1 \\
\hline Q6ZYK7 & PTGS2 & Prostaglandin G/H synthase 2 \\
\hline X6RJD6 & PTGS1 & Prostaglandin G/H synthase 1 \\
\hline P19793 & RXRA & etinoic acid receptor RXR-alpha \\
\hline P35368 & ADRA1B & Alpha-1B adrenergic receptor \\
P06400 & RB1 & Retinoblastoma-associated protein \\
P08588 & ADRB1 & Beta-1 adrenergic receptor \\
Q01094 & E2F1 & Transcription factor E2F1 \\
Q14524 & SCN5A & Sodium channel protein type 5 subunit alpha \\
P61925 & PKIA & cAMP-dependent protein kinase inhibitor alpha \\
P01344 & IGF2 & Insulin-like growth factor II \\
A0A0U1RRB2 & GABRA1 & Gamma-aminobutyric acid receptor subunit alpha-1 \\
\hline
\end{tabular}

\section{Figures}



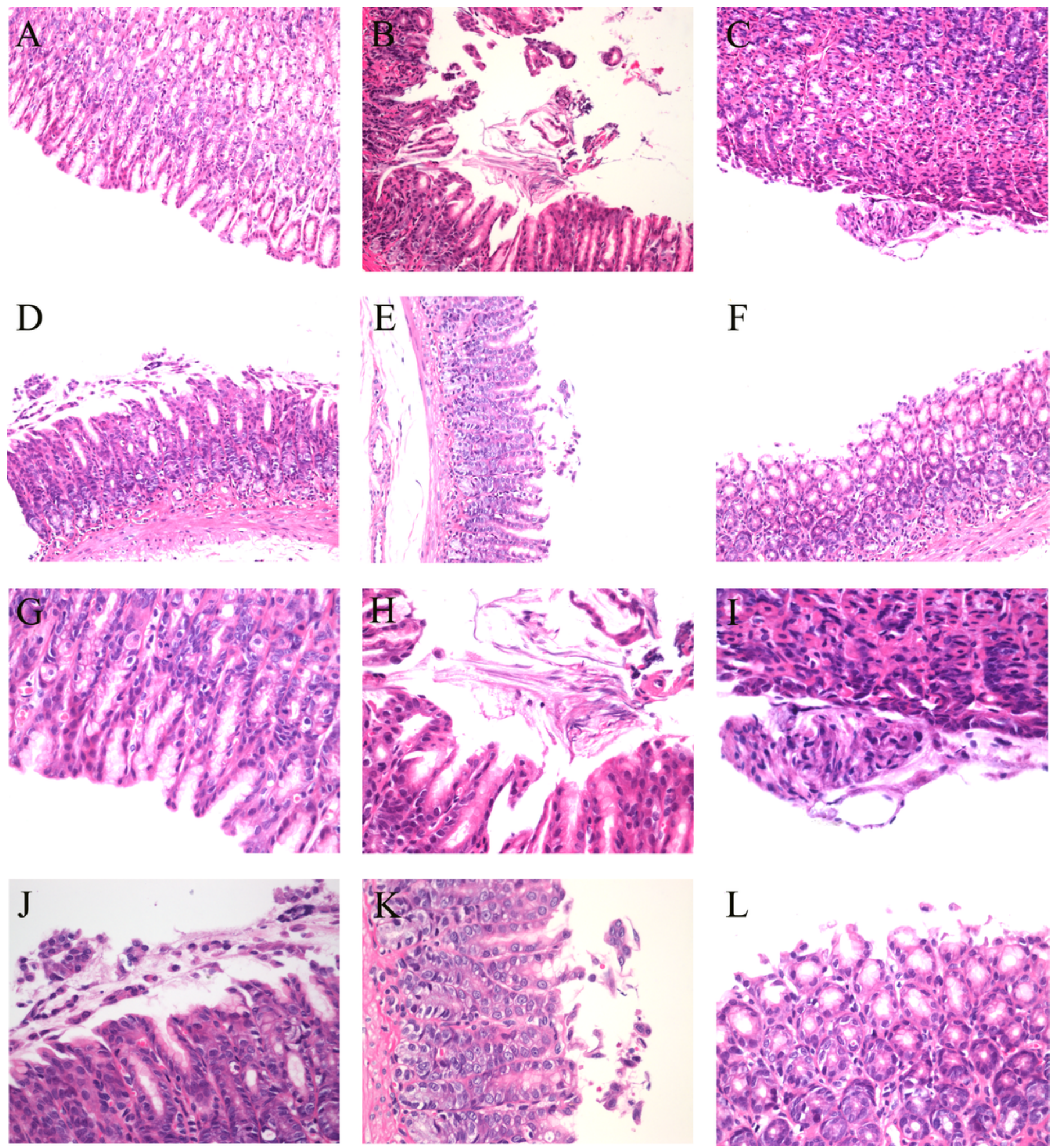

Figure 1

ZJP affects gastric histopathology in CAG rats. (A) Control group; (B) Model group; (C) $0.63 \mathrm{~g} / \mathrm{kg}$ ZJP group; (D) $1.26 \mathrm{~g} / \mathrm{kg}$ ZJP group; (E) $2.52 \mathrm{~g} / \mathrm{kg}$ ZJP group; (F) omeprazole group (HE staining, $\times 200$ magnification); (G) Control group; (H) Model group; (I) $0.63 \mathrm{~g} / \mathrm{kg}$ ZJP group; (J) $1.26 \mathrm{~g} / \mathrm{kg}$ ZJP group; (K) $2.52 \mathrm{~g} / \mathrm{kg}$ ZJP group; (L) omeprazole group (HE staining, $\times 400$ magnification). 

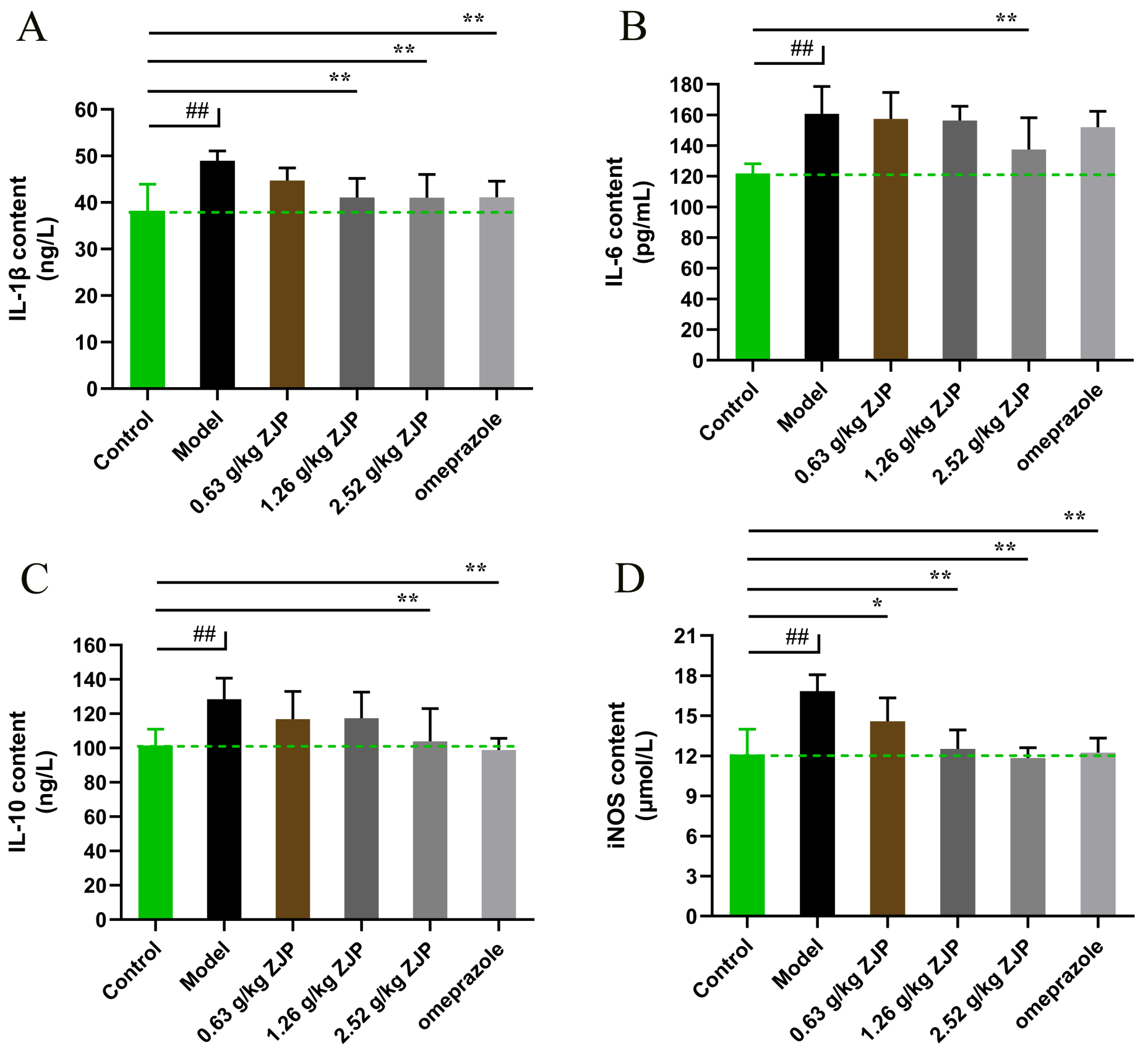

Figure 2

ZJP affects serum biochemical parameters in CAG rats (mean $\pm S D, n=6$ ). (A) IL-1 $1 \beta$ content; (B) IL-6 content; (C) IL-10 content; (D) iNOS content. \#\#P< 0.01, compared with the control group. ${ }^{*} P<0.05$, $* * P<$ 0.01 , compared with the model group. 

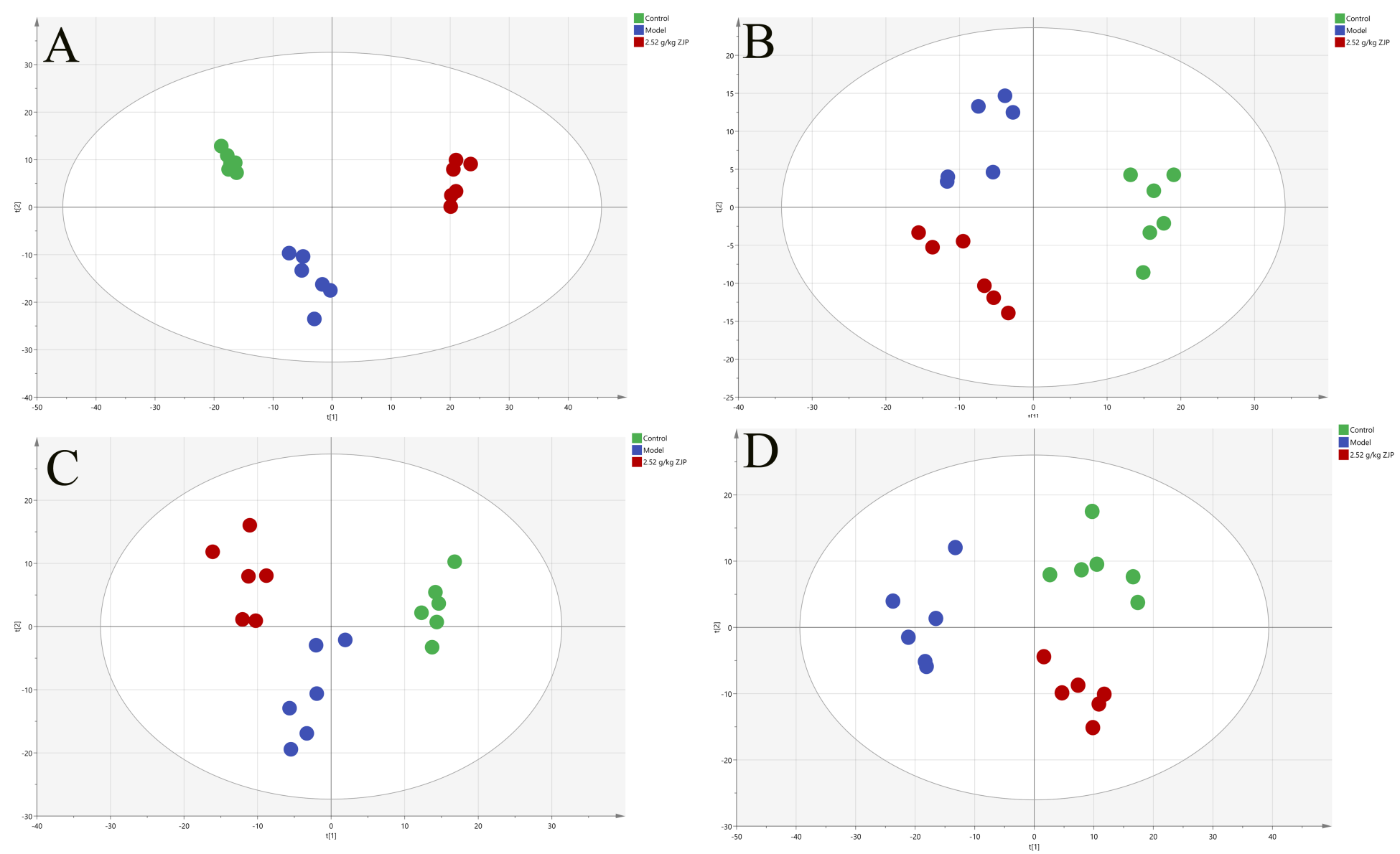

Figure 3

PCA score plot of the control group, model group and $2.52 \mathrm{~g} / \mathrm{kg}$ ZJP group. (A) ESI+ mode of PCA in serum samples between the three groups; (B) ESI- mode of PCA in serum samples between the three groups; (C) ESI+ mode of PCA in urine samples between the three groups; (D) ESI- mode of PCA in urine samples between the three groups. 


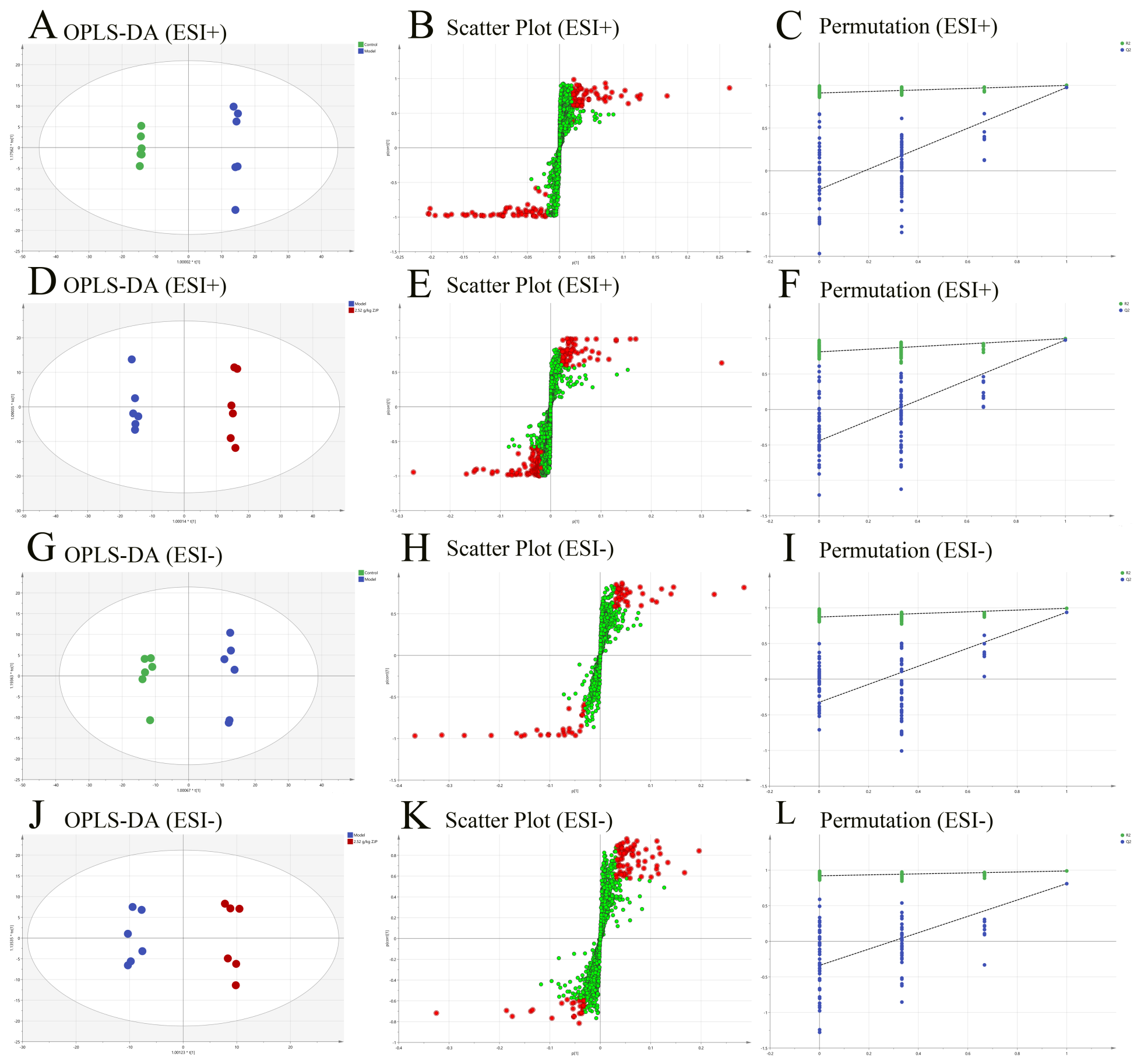

\section{Figure 4}

The OPLS-DA score plots, S-plots and 100-permutation test generated of serum samples between the control group, model group and $2.52 \mathrm{~g} / \mathrm{kg}$ ZJP group. OPLS-DA score plots were the pair-wise comparisons in ESI+ mode, control group vs model group (A), model group vs $2.52 \mathrm{~g} / \mathrm{kg}$ ZJP group (D) as well as in ESI- mode, control group vs model group (G), model group vs $2.52 \mathrm{~g} / \mathrm{kg}$ ZJP group (J); S-plots of the OPLS-DA model in ESI+ mode, control group vs model group (B), model group vs $2.52 \mathrm{~g} / \mathrm{kg} Z \mathrm{JP}$ group (E) as well as in ESI- mode, control group vs model group $(\mathrm{H})$, model group vs $2.52 \mathrm{~g} / \mathrm{kg}$ ZJP group (K); The 100-permutation test of the OPLS-DA model in ESI+ mode, control group vs model group (C), 
model group vs $2.52 \mathrm{~g} / \mathrm{kg}$ ZJP group (F) as well as in ESI- mode, control group vs model group (I), model group vs $2.52 \mathrm{~g} / \mathrm{kg}$ ZJP group (L).
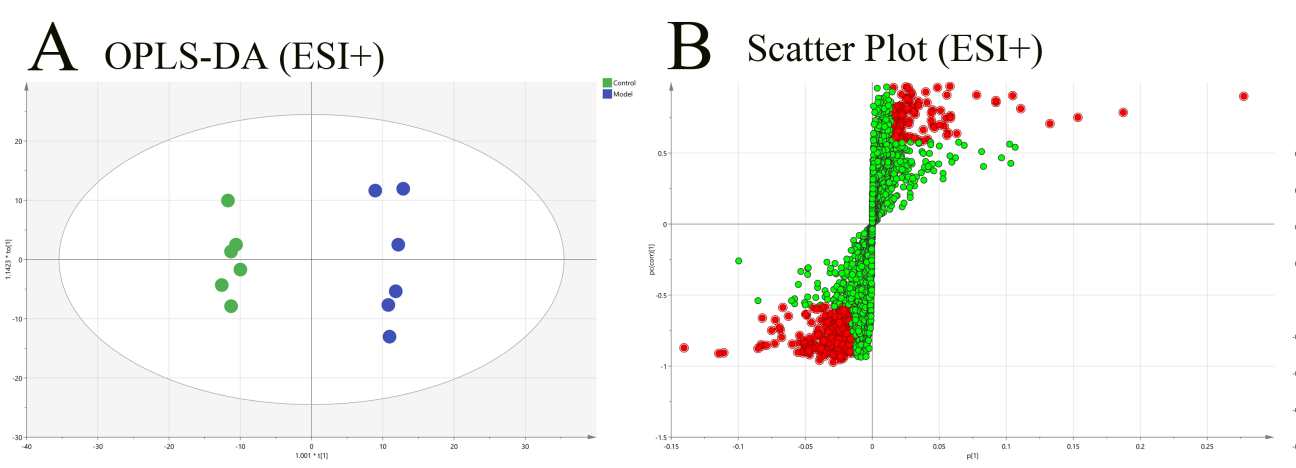

C Permutation (ESI+)
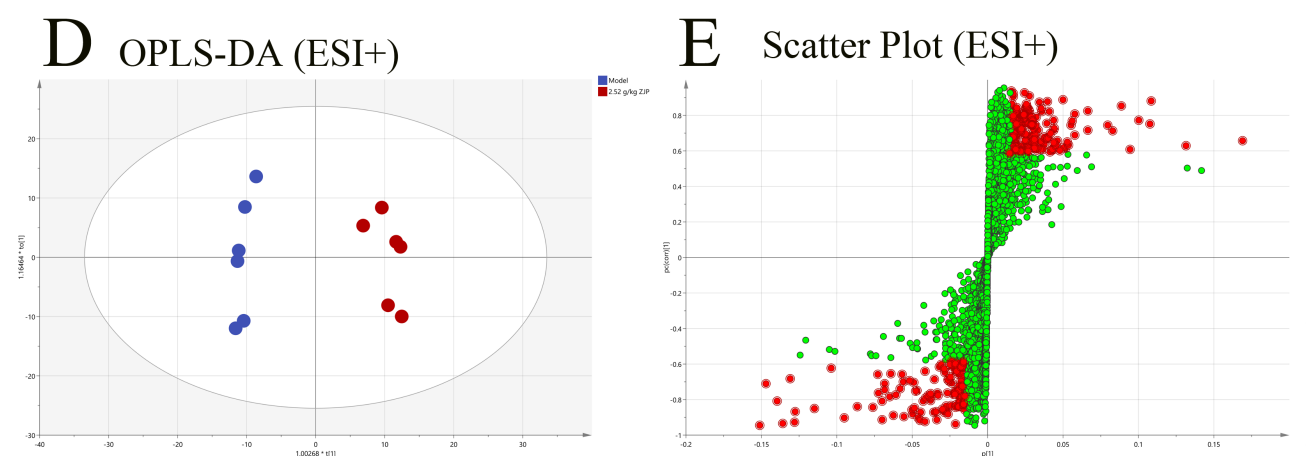

F Permutation (ESI+)
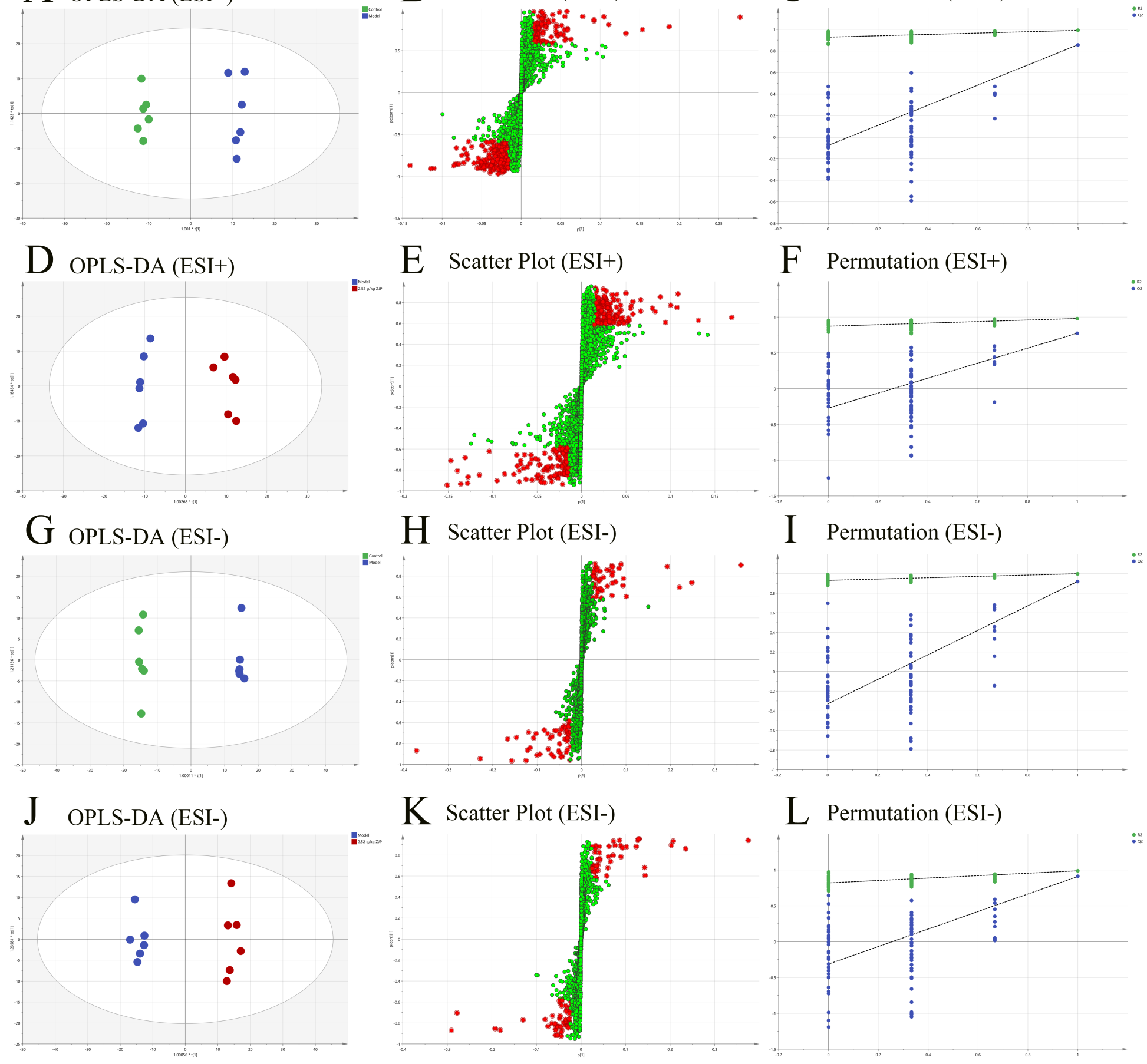

\section{Figure 5}

The OPLS-DA score plots, S-plots and 100-permutation test generated of urine samples between the control group, model group and $2.52 \mathrm{~g} / \mathrm{kg}$ ZJP group. OPLS-DA score plots were the pair-wise comparisons in ESI+ mode, control group vs model group (A), model group vs $2.52 \mathrm{~g} / \mathrm{kg}$ ZJP group (D) as well as in ESI- mode, control group vs model group (G), model group vs $2.52 \mathrm{~g} / \mathrm{kg}$ ZJP group (J); S-plots of the OPLS-DA model in ESI+ mode, control group vs model group (B), model group vs $2.52 \mathrm{~g} / \mathrm{kg}$ ZJP 
group (E) as well as in ESI- mode, control group vs model group (H), model group vs $2.52 \mathrm{~g} / \mathrm{kg}$ ZJP group (K); The 100-permutation test of the OPLS-DA model in ESI+ mode, control group vs model group (C), model group vs $2.52 \mathrm{~g} / \mathrm{kg}$ ZJP group (F) as well as in ESI- mode, control group vs model group (I), model group vs $2.52 \mathrm{~g} / \mathrm{kg}$ ZJP group (L).

A

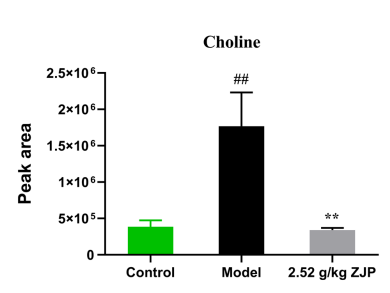

$\mathrm{D}$

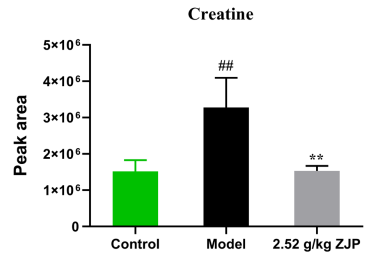

G

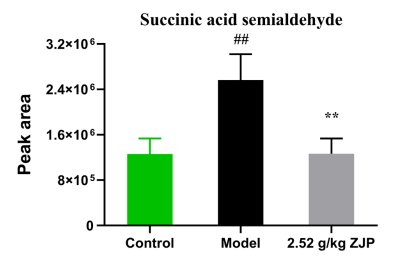

$\mathrm{J}$

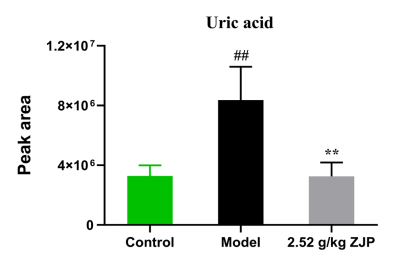

$\mathrm{B}$

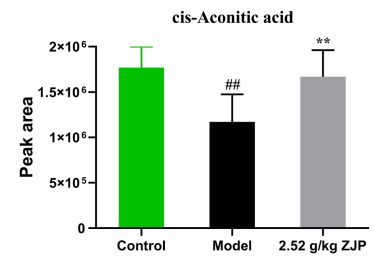

$\mathrm{E}$

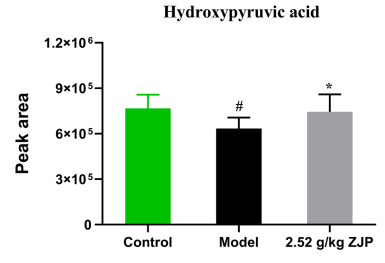

$\mathrm{H}$

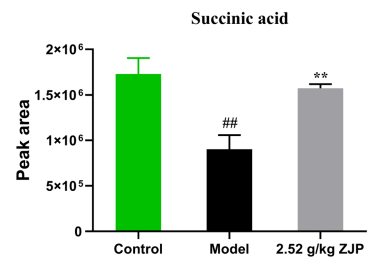

C

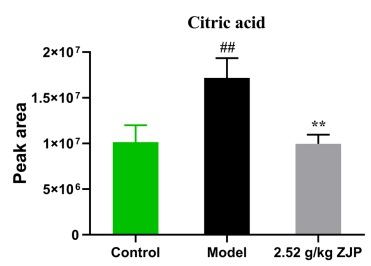

$\mathrm{F}$

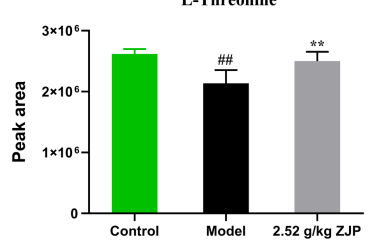

I

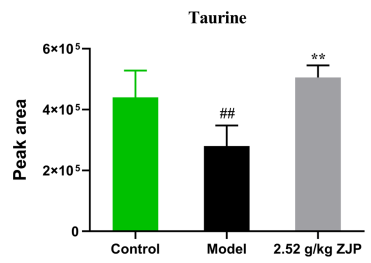

\section{Figure 6}

Potential metabolites changes in H. pylori-induced CAG with ZJP treatment. (A) Choline; (B) cis-Aconitic acid; (C) Citric acid; (D) Creatine; (E) Hydroxypyruvic acid; (F) L-Threonine; (G) Succinic acid semialdehyde; $(\mathrm{H})$ Citric acid; (I) Taurine; $(\mathrm{J})$ Uric acid. \#P $<0.05$ and \#\#P $<0.01$, compared with the control group. ${ }^{\star} \mathrm{P}<0.05,{ }^{\star} \mathrm{P}<0.01$, compared with the model group. 

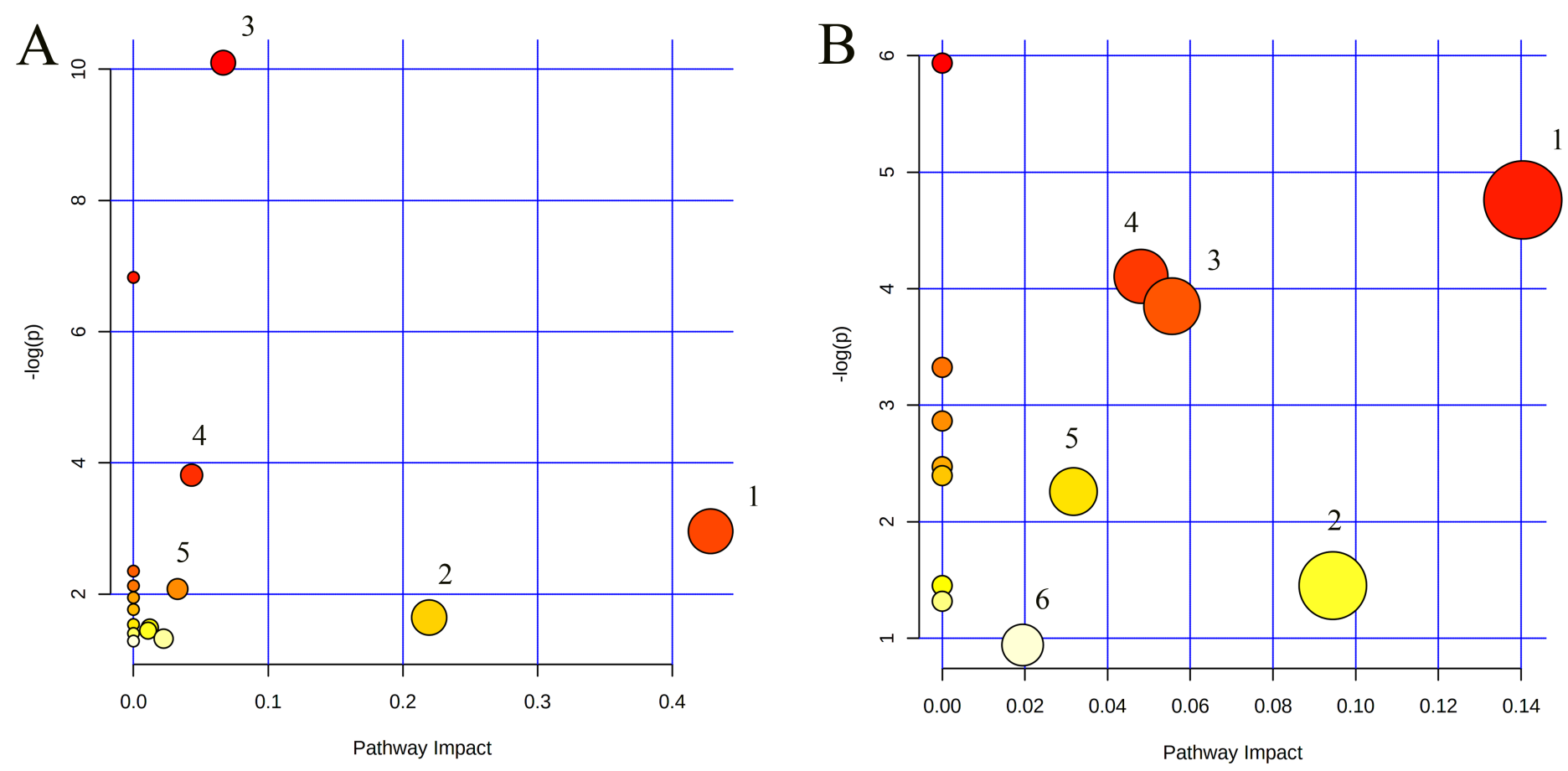

Figure 7

Summary of metabolic pathway analysis of potential metabolites. (A) Summary of metabolic pathway analysis of potential metabolites in serum samples; 1 . Taurine and hypotaurine metabolism; 2 . Glyoxylate and dicarboxylate metabolism; 3. Glycine, serine and threonine metabolism; 4. Glycerophospholipid metabolism; 5. TCA cycle. (B) Summary of metabolic pathway analysis of potential metabolites in urine samples. 1. TCA cycle; 2. Glycerophospholipid metabolism; 3. Glyoxylate and dicarboxylate metabolism; 4. Alanine, aspartate and glutamate metabolism; 5. Butanoate metabolism; 6. Purine metabolism.
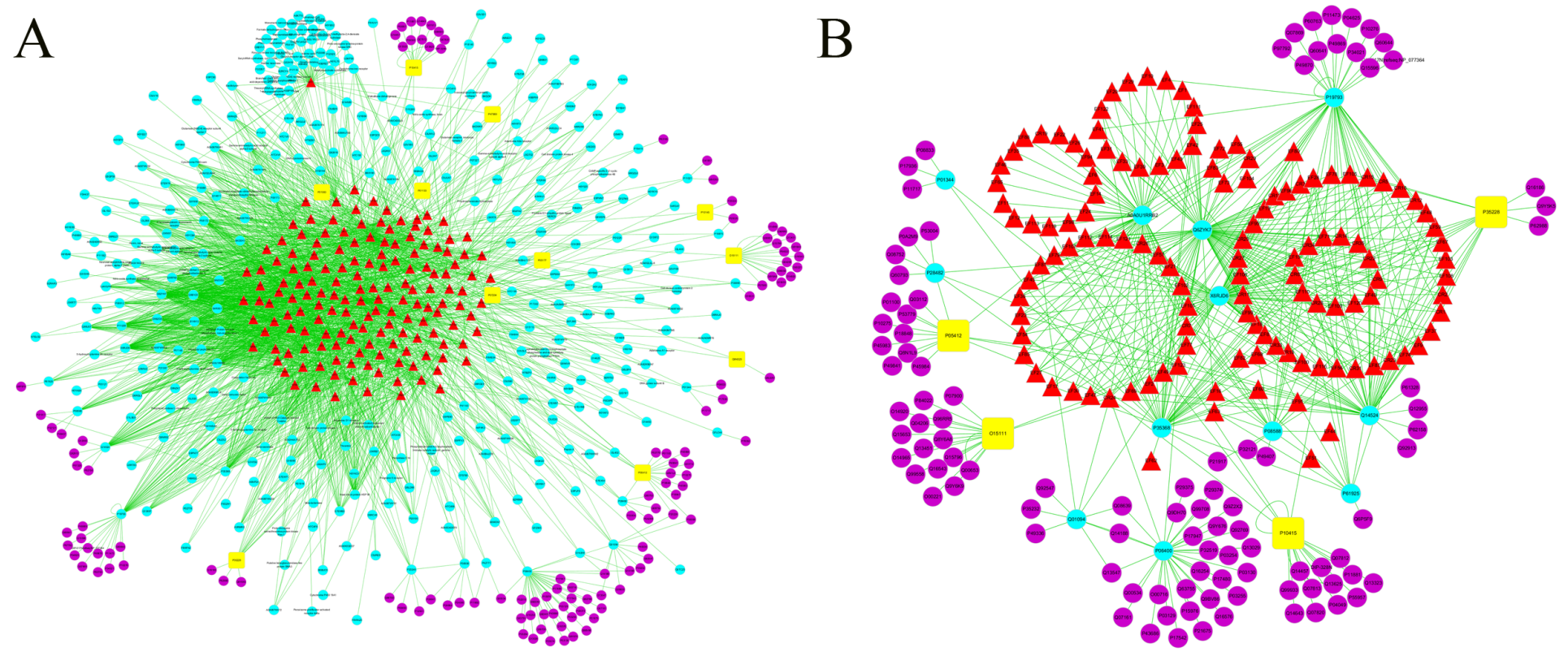

Figure 8 
The crucial compound-CAG target-metabolite network. (A) All target information participating in the treatment of CAG by ZJP; (B) key target information participating in the treatment of CAG by ZJP. The red triangle represents the active chemical composition of ZJP; the blue dots represent the protein targets of ZJP; the yellow square represents the common target for ZJP and disease; purple dots represent targets associated with potential metabolites.
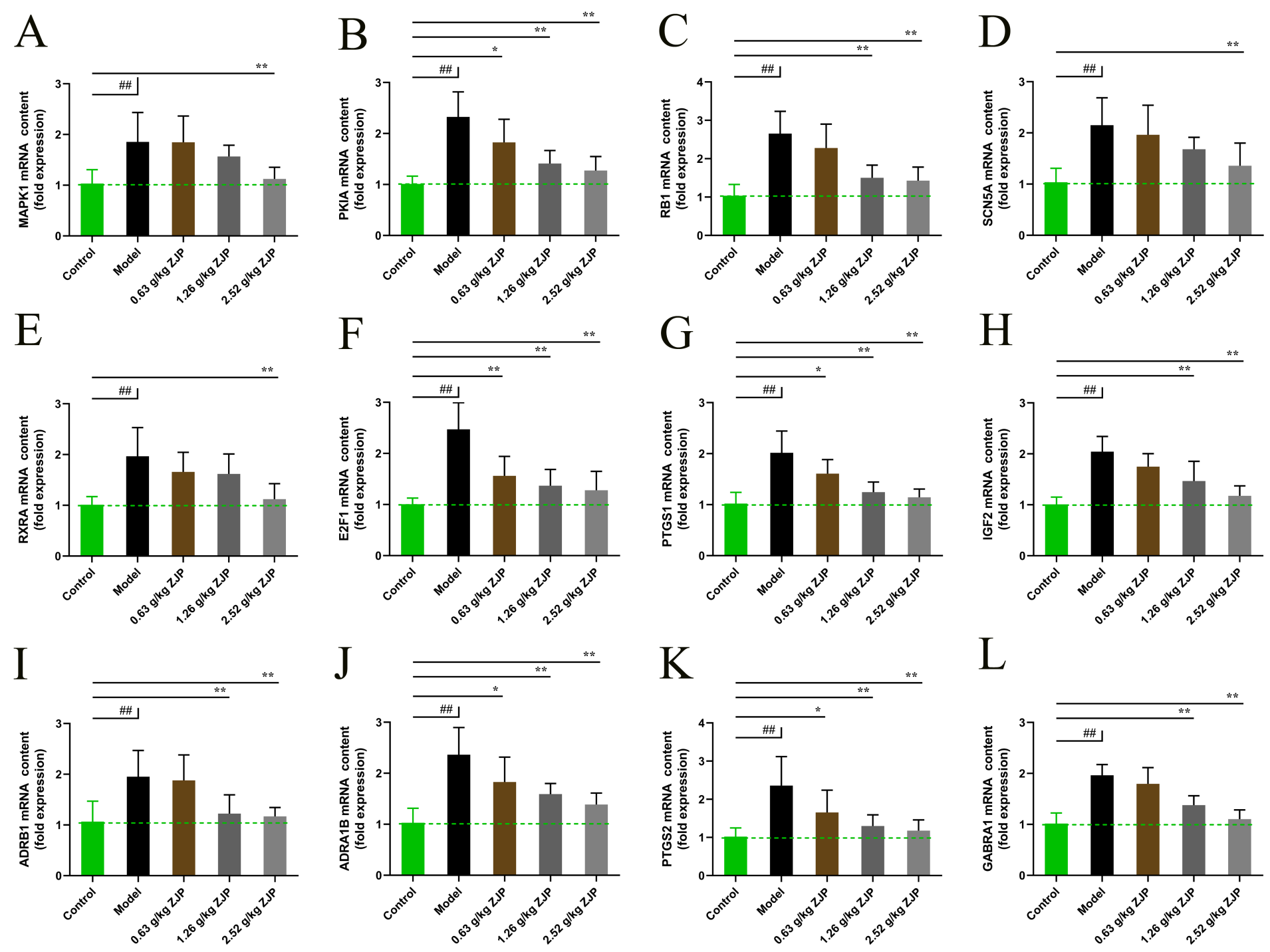

$\mathrm{L}$

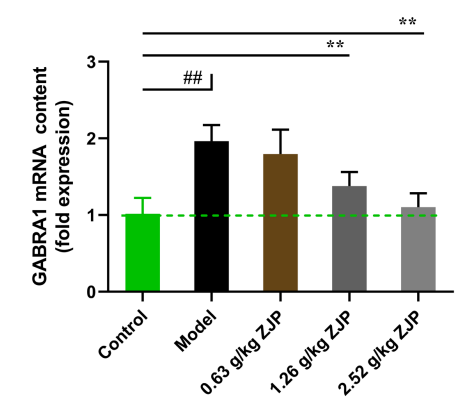

Figure 9

Effect of ZJP on the relative mRNA expressions of MAPK1, PKIA, RB1, SCN5A, RXRA, E2F1, PTGS1, IGF2, ADRB1, ADRA1B, PTGS2, and GABRA1 in rats with CAG. (A) MAPK1; (B) PKIA; (C) RB1; (D) SCN5A; (E) RXRA; (F) E2F1; (G) PTGS1; (H) IGF2; (I) ADRB1; (J) ADRA1B; (K) PTGS2; (L) GABRA1. \#\#P < 0.01, compared with the control group. ${ }^{\star} P<0.05,{ }^{*} \mathrm{P}<0.01$, compared with the model group. 